\title{
Study of the microstructure and pores distribution of lightweight mortar containing polymer waste aggregates
}

\author{
V. Calderón ${ }^{1}, \mathrm{~S}$. Gutiérrez-González ${ }^{1}$, A. Rodriguez ${ }^{1}$ \\ $\&$ M. Horgnies ${ }^{2}$ \\ ${ }^{1}$ Departamento de Construcciones Arquitectónicas e Ingenierías de la \\ Construcción y del Terreno, Universidad de Burgos, Spain \\ ${ }^{2}$ Lafarge Centre de Recherche, Saint Quentin-Fallavier, France
}

\begin{abstract}
The study of mortars containing polymer wastes is a key-topic to create new construction materials for a sustainable development. This work deals with the characterization of the microstructure of lightweight mortars containing polymer waste powder, which are recycled, such as aggregates. The observations of crosssections and fractured samples using Scanning Electron Microscopy (SEM) highlight that the particles of polymer are homogeneously dispersed into the mortar matrix. Numerous capillarity pores are detected into the hydrated cement paste. Calcium Silicate Hydrates covering the particles of polymer are also detected in environmental mode. The porosity is analyzed using two complementary techniques: (i) Mercury Intrusion Porosimetry (MIP) revealing the micro-pores sized from 170-200 $\mu \mathrm{m}$ up until 5-10 nm; and (ii) X-ray computerized axial tomography providing the macro-pores sized larger than $170 \mu \mathrm{m}$. The distribution of the total intruded volume is quantified by MIP according to the ratio sand/particles of polymer. The distortion of the pores distribution increases as a function of the content of polymer residues, excepted when the particles of polymer are replacing $25 \%$ of sand. In this specific case, the measurements by MIP and tomography show a homogeneous distribution of the larger pores, close to the one of the reference mortar.
\end{abstract}

Keywords: lightweight mortar, waste polymer, microstructure, tomography, MIP, SEM. 


\section{Introduction}

New construction materials and an efficient recycling of polymer-based wastes are both expected to promote a sustainable development. Numerous studies have been performed on mortars and concretes containing polymer particles or waste polymer residues to obtain improved properties in terms of thermal or acoustic insulation [1-4]. The relations between the final porosity and the properties of hardened mortars were studied by several authors. Bouvard et al. [5] investigated the microstructure of lightweight concrete containing millimeter-size expanded polystyrene spheres by X-ray tomography. Following previous studies $[6,7]$ dealing with the substitution of sand by residues of polyurethane foam, this work focuses on the characterization of hardened mortars containing waste polyamide powder. First, the interface between the particles of polymer and the hydrated cement paste is investigated by SEM and Energy-Dispersive X-ray spectroscopy (EDS). Second, the porosity of hardened mortars is measured using two complementary techniques: MIP and X-ray tomography, which provide results about the micro-porosity and macro porosity, respectively.

\section{Material and methods}

\subsection{Materials}

\subsubsection{Polymer Waste Particles (PWP)}

Particles made of polyamide were obtained from waste raw material generated in an industrial Laser Sintering process. Its granulometric size was determined through laser diffraction analysis using a HELOS $12 \mathrm{kv}$ SYMPATEC analyzer (as shown in Figure 1). The real density of the PWP is $1.07 \mathrm{~g} / \mathrm{cm}^{3}$, which was measured with the Pycnometer method, using isopropyl alcohol [8].

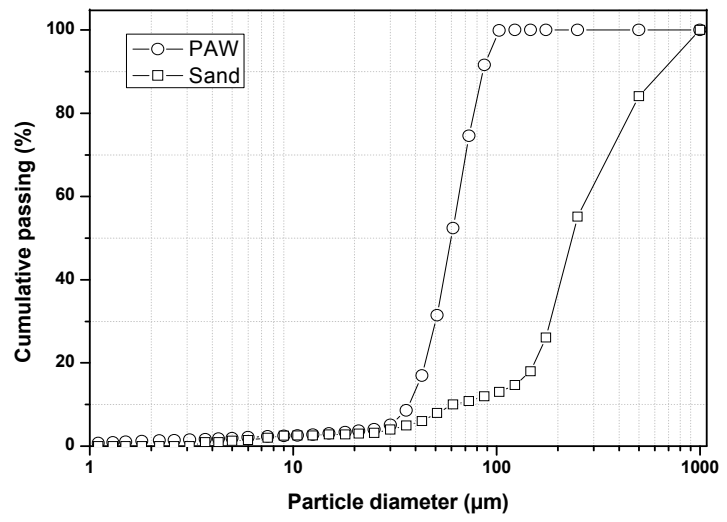

Figure 1: $\quad$ Laser granulometry of the polymer waste particles and sand. 


\subsubsection{Lightweight mortar samples}

The mortar mix-design was traditional, containing cement, sand and water. Ordinary Portland cement (type CEM I $42.5 \mathrm{R}$ ) with a density of $3065 \mathrm{~kg} / \mathrm{m}^{3}$ and river sand, sieved between 0 and $2 \mathrm{~mm}$ with a density of $2600 \mathrm{~kg} / \mathrm{m}^{3}$, were used. The lightweight mortar samples were manufactured by replacing different percentages of sand with the polymer waste particles. The particle size distribution of river sand is compared to the one of the PWP in Figure 1.

The lightweight mortar was produced using a 1/3 relation cement/(sand + PWP). Although the initial dosages were considered by weight, the sand was substituted by an equivalent quantity of polymer particles in volume (Table 2). The amount of water was adjusted to ensure good workability, fluency and plastic state, in accordance with Standard EN 1015-3 [9].

Fresh density and occluded air in fresh mortar samples were measured at $20( \pm 1)^{\circ} \mathrm{C}$ and $50( \pm 1) \%$ of relative humidity, according to the standards [10, 11]. The occluded air increases according to the amount of polymer waste particles introduced into the mix (replacing the sand) except for the P25 and P50 samples, which show respectively a minor and a similar content of occluded air compared to the one of the reference sample. As expected by the intrinsic low density of the polyamide waste, the fresh density of the samples decreases proportionally as a function of the replacement of sand by the particles of polymer.

Table 1: $\quad$ Mix proportions and properties of the mortar samples.

\begin{tabular}{|c|c|c|c|c|}
\hline Samples & $\begin{array}{c}\text { Sand replaced by PWP } \\
\text { (\% in volume) }\end{array}$ & Water/Cement & $\begin{array}{c}\text { Fresh density } \\
\left(\mathrm{kg} / \mathrm{m}^{3}\right)\end{array}$ & $\begin{array}{c}\text { Occluded } \\
\text { air (\%) }\end{array}$ \\
\hline R & 0 & 0.71 & 2123 & 4.7 \\
\hline P25 & 25 & 0.74 & 1910 & 4.2 \\
\hline P50 & 50 & 0.75 & 1690 & 4.5 \\
\hline P75 & 75 & 0.77 & 1440 & 6.3 \\
\hline P100 & 100 & 0.89 & 1200 & 7.4 \\
\hline
\end{tabular}

\subsection{Experimental methods}

\subsubsection{SEM and EDS}

Selected mortar sample was cut, impregnated in resin and then polished before being characterized in Back Scattering Electron Diffraction (accelerating voltage of $15 \mathrm{keV}$; current intensity of $1 \mathrm{nA}$ ), using a high-resolution field-effect gun scanning electron microscope (SEM FEG Quanta 400 from FEI Company, USA). EDS spectrometry was used to map the distribution of specific elements such as carbon and chlorine, which characterized the polished cross-section. Certain fractured specimens were not polished and were analyzed in environmental mode using a pressure of 3.2 $\mathrm{Pa}$ (to limit the dehydration). 


\subsubsection{MIP}

The porosity of mortar samples was investigated by MIP technique (Autopore IV from Micromeritics, USA). The pressure range of the porosimeter was from sub ambient up to $400 \mathrm{MPa}$, covering the pore diameter range from about $360 \mu \mathrm{m}$ to

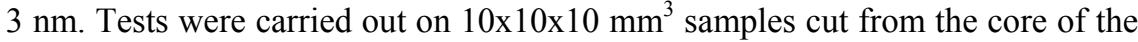
mortar. The samples were dried in an oven at $45^{\circ} \mathrm{C}$ overnight before being tested. The advancing/receding contact angle was assumed to be $130^{\circ}$, as recommended for ordinary cement pastes [12].

\subsubsection{X-ray computerized axial tomography}

$\mathrm{X}$-ray tomography allows the non-invasive characterization of the microstructure of numerous types of materials evaluation [13, 14], including concrete [15]. The mortars were cut and drilled after being cured to obtain cylindrical samples. The scans were made with a transmission type X-ray tube Yxlon working at $225 \mathrm{Kv} / 30 \mathrm{~mA}$. The sample was placed on a platform rotating $360^{\circ}$ with a step of 0.3 . A 2D scintillator panel detector of $2048 \times 2048$ pixels was used to record the images of the samples to reconstruct the internal structure. In these conditions, 1200 projections were taken for each sample and the data were reconstructed into 1-CT slices using Mimics 10.0 software.

\section{Results}

\subsection{Observations by SEM and EDS spectrometry}

Figure 2 shows a SEM image of a P50 cross-section: numerous particles of polymer are dispersed into the hydrated paste. Numerous small pores (related to the large capillarity pores) are also detected into the hydrated cement paste at high magnification.

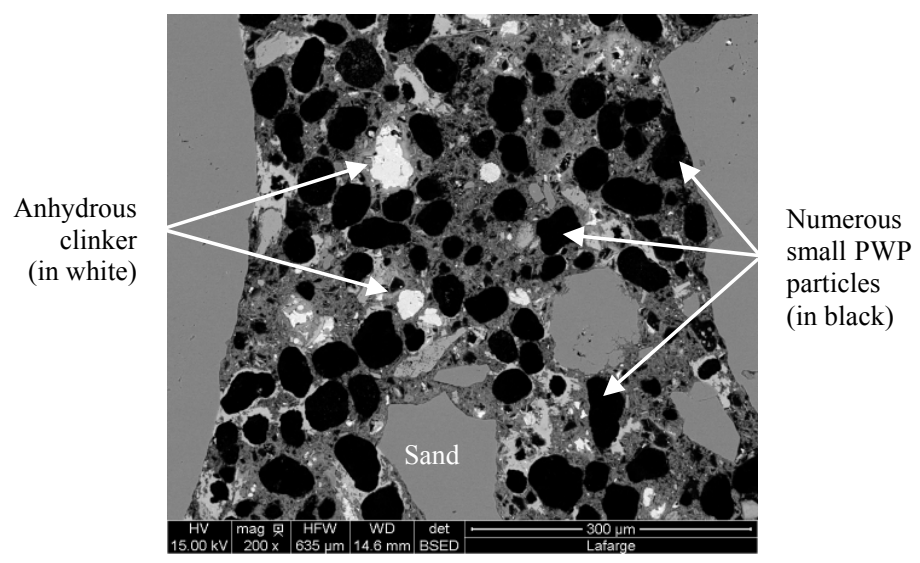

Figure 2: $\quad$ Polished cross-section of the P50 sample observed by SEM. 
Figure 3 provides a map acquired by EDS spectrometry, showing the elemental composition of a P50 cross-section. Carbon and chlorine elements are then represented in red and green, respectively. Because chlorine is assigned to the epoxy resin used to impregnate the mortar samples before the polishing, the areas completely green can be assigned to the presence of air voids. Carbon is mainly related to the particles of polymer, which are homogeneously dispersed into the cement paste surrounding the sand aggregates (darker areas).

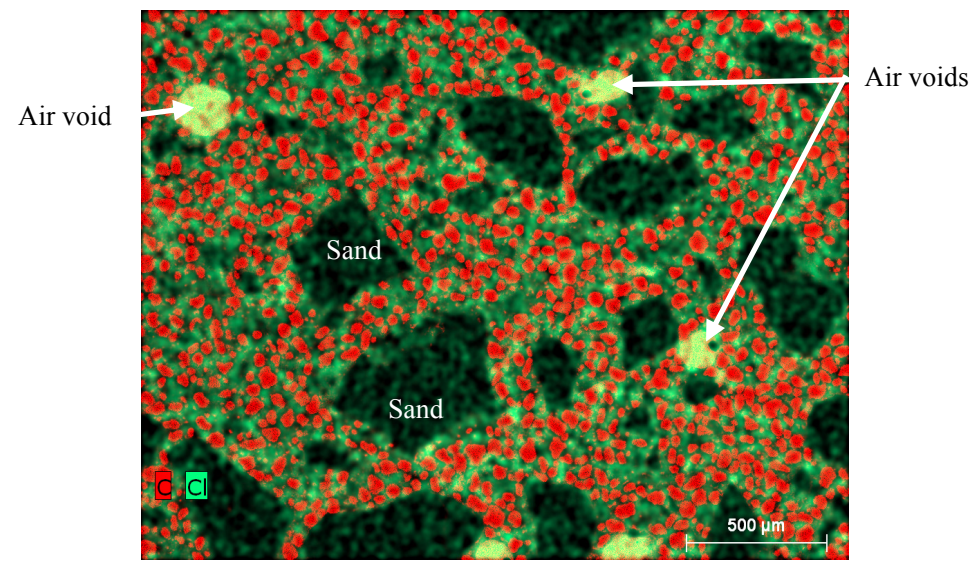

Figure 3: Distribution of carbon (red) and chlorine (green) for a P50 crosssection (acquired by EDS spectrometry).

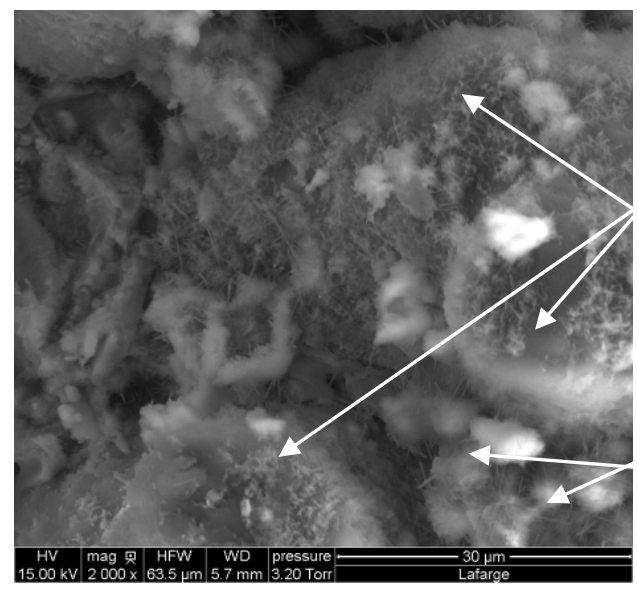

PWP particles covered by numerous $\mathrm{C}-\mathrm{S}-\mathrm{H}$

C-S-H of the hydrated paste located between the PWP particles

Figure 4: $\quad$ SEM images of C-S-H covering the particles of polymer (P50 sample).

Figure 4 provides a SEM image of the fractured P50 sample acquired in environmental mode: Calcium Silicate Hydrates (C-S-H) are covering the particles of polymer. Moreover, a large amount of C-S-H and some crystals of 
portlandite are detected into the paste located between the particles confirms that the recycled polymer does not disturb the hydration of cement paste. No crystals of ettringite are highlighted by the SEM observations.

\subsection{Study of the pores distribution using MIP}

Figure 5 shows the differential distribution curves, according to the ratio of sand replaced by the particles of polymer. These curves were plotted to better estimate the critical pore diameter that corresponds to the maximum peaks in the distribution, above which no connected path could form throughout the sample [16]. The distribution of the pore diameter into the mortar samples becomes more and more disturbed when more than $50 \%$ of sand is replaced by the PWP. However, the differential distribution curve of P25 sample is close to the one of the reference mortar sample. These results are conformed to the values of occluded air measured in the fresh mortar mixes.

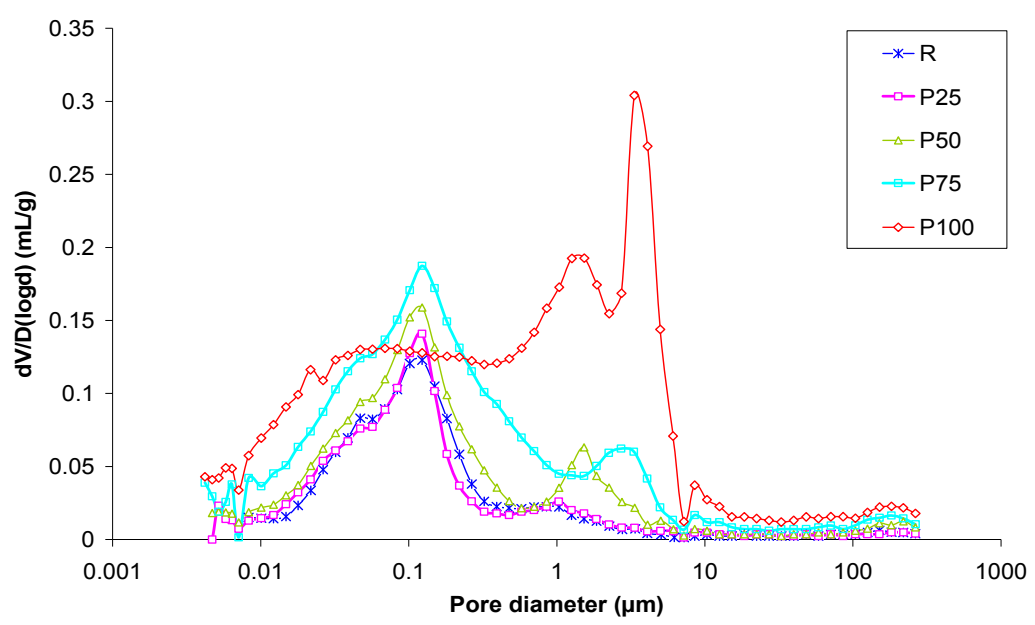

Figure 5: Differential distribution curves according to the ratio of sand replaced by the PWP particles.

Table 1 provides the distributions of the total intruded volume for the different mortar samples. The pore sizes are classified in 4 distinct ranges [17, 18]: (i) $\mathrm{d}>10 \mu \mathrm{m}$ : air voids; (ii) $0.05<\mathrm{d}<10 \mu \mathrm{m}$ : large capillarities; (iii) $0.01<\mathrm{d}<0.05 \mu \mathrm{m}$ : medium capillarities; (iv) $<0.01 \mu \mathrm{m}$ : small capillarities. The pore structure is more and more disturbed as a function of the substitution of sand by the particles of polymer (due to the increase of large capillarities and air voids), excepted for the P25 sample that shows a porosity distribution close to the one of the reference sample, confirming the previous results of Figure 5. 
Table 2: $\quad$ Distribution of total intruded volume of the mortar samples.

\begin{tabular}{|c|c|c|c|c|c|}
\hline \multirow{2}{*}{$\begin{array}{c}\text { Range of } \\
\text { porosity }\end{array}$} & \multicolumn{5}{|c|}{ Mortar samples (ratio of sand replaced by the PWP particles) } \\
\cline { 2 - 6 } & $\mathrm{R}$ & $\mathrm{P} 25$ & $\mathrm{P} 50$ & $\mathrm{P} 75$ & $\mathrm{P} 100$ \\
\hline$<0.01 \mu \mathrm{m}$ & 6.5 & 4.3 & 3.8 & 4.0 & 4.3 \\
\hline $0.01-0.05 \mu \mathrm{m}$ & 22.7 & 25.1 & 21.5 & 20.9 & 17.3 \\
\hline $0.05-10 \mu \mathrm{m}$ & 65.8 & 65.6 & 69.0 & 68.2 & 71.0 \\
\hline$>10 \mu \mathrm{m}$ & 4.9 & 5.0 & 5.7 & 6.9 & 7.5 \\
\hline
\end{tabular}

\subsection{Study of the pores distribution using X-rays tomography}

Based on the computational analysis of X-rays images, tomography allows characterizing the internal structure of the lightweight mortar samples. Figure 6 provides all the axial sections recorded during the study. The comparison between all the images allows mapping the distribution of the different materials (pores, cement, PWP and sand) according to the ratio of sand replaced by the polymer particles.

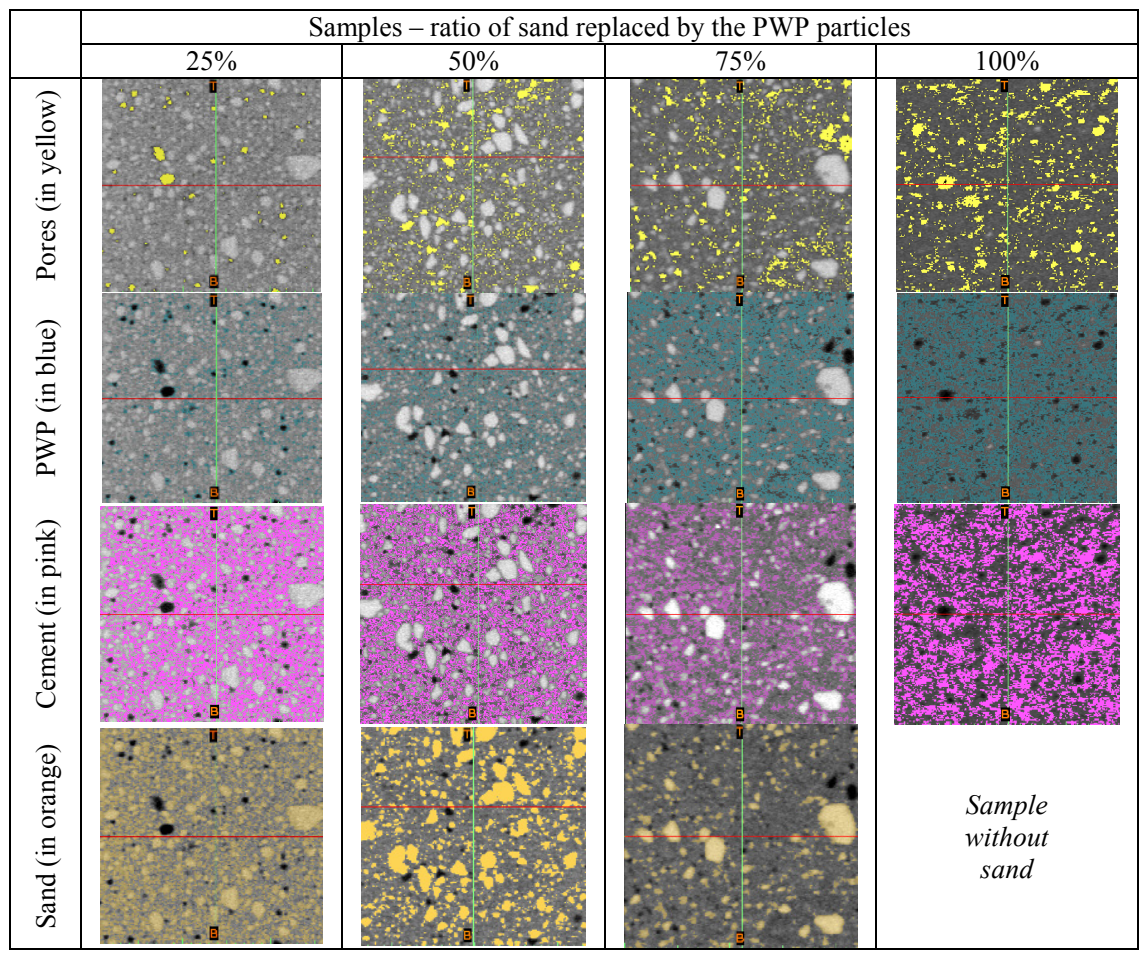

Figure 6: Tomography sections of the materials contained in the mortar samples. 
As shown in Figure 7 and using the sequence of images dedicated to the pores distribution, it is possible to reconstruct the $3 \mathrm{D}$ images of the macro-porosity (pores sizes $>170 \mu \mathrm{m}$ ) according to the ratio of sand replaced by the polymer particles.

\begin{tabular}{|c|c|c|c|}
\hline \multicolumn{5}{|c|}{ Mortar samples (ratio of sand replaced by the PWP particles) } \\
\hline P25 & P50 & P75 & P100 \\
\hline & & & \\
\hline
\end{tabular}

Figure 7: 3D tomography images of the pores distribution for each mortar sample containing PWP particles.

Figure 8(a) shows an example of axial section of the distribution of materials composing the mortar sample when the PWP particles are replacing $25 \%$ of sand. Each color represents one specific material: (i) blue for the polyamide particles; (ii) pink for the cement paste; (iii) orange for the natural sand aggregates and (iv) yellow for the pores. Figure 8(b) shows an example of 3D reconstruction done on the same samples.
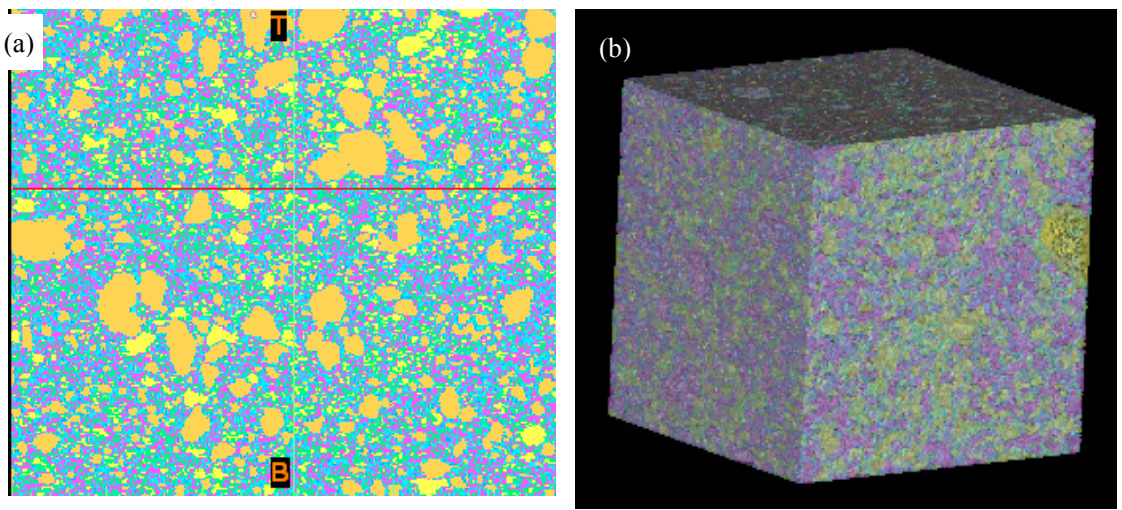

Figure 8: $\quad$ P25 mortar sample: (a) section of the materials distribution; (b) 3D reconstruction of the mortar sample.

\section{Conclusions}

Lightweight mortars can be manufactured using a high ratio of polymer waste particles (made of recycled polyamide) in place of sand. The investigation of the microstructure by several methods of characterization (SEM, EDS spectroscopy, MIP and X-ray tomography) allows obtaining different data. 
(i) The polymer waste particles are homogeneously dispersed into the hydrated cement paste;

(ii) The composition of the polymer waste particles does not disturb the hydration of the cement paste (numerous $\mathrm{C}-\mathrm{S}-\mathrm{H}$ are detected);

(iii) The addition of polymer waste particles into the mortar mix increases the amounts of air voids and large capillarities pores;

(iv) The microstructure and the pores distribution are significantly disturbed when more than $50 \%$ of sand is replaced by the polymer waste particles;

(v) The replacement of $25 \%$ of sand by the polymer waste particles provides a microstructure and a pore distribution close to those of a reference sample.

\section{References}

[1] Siddique, R., Khatib, J. and Kaur, I., Use of recycled plastic in concrete: A review. Cement and Concrete Composites, 28, pp. 1835-1852, 2008.

[2] Peng, J.H., Chen, M.F. and Zhang, J.X., Study on waste expanded polystyrene as lightweight aggregate for thermal insulating mortar. Journal of Building Materials, 5, pp. 166, 2002.

[3] Wang, R. and Meyer, C., Performance of cement mortar made with recycled high impact polystyrene. Cement and Concrete Composites, 34, pp. 975-981. 2012.

[4] Choi Y-W. and Moon D-J., Chung J-S., Cho S-K., Effects of waste PET bottles aggregate on the properties of concrete. Cement and Concrete Research, 35, pp. 776-781, 2005.

[5] Bouvard, D., Chaix, J.M., Dendievel, R., Fazekas, A., Létang, J.M., Peix, G. and Quenard, D., Characterization and simulation of microstructure and properties of EPS lightweight concrete. Cement and Concrete Research, 37, pp. 1666-1673, 2007.

[6] Gadea, J., Rodriguez A., Campos, P.L., Garabito, J. and Calderon, V., Lightweight mortar made with recycled polyurethane foam, Cement and Concrete Composites, 32, pp. 672-677, 2010.

[7] Junco, C., Gadea, J., Rodriguez, A., Gutierrez-Gonzalez, S. and Calderon, V., Durability of lightweight masonry mortars made with white recycled polyurethane foam, Cement and Concrete Composites, 34, pp. 1174-1179, 2012.

[8] EN 1936. Natural stone test methods. Determination of real density and apparent density and of total and open porosity; 2006.

[9] EN 1015-3. Methods of test for mortar for masonry. Part 3: Determination of consistence of fresh mortar (by flow table). 1999.

[10] EN 1015-6. Methods of test for mortar for masonry. Part 6: Determination of occluded air. 1999.

[11] EN 1015-10. Methods of test for mortar for masonry - Part 10. Determination of dry bulk density of hardened mortar; 2000.

[12] Taylor, H.F.W., Cement Chemistry, Thomas Telford Publishing, London, $2^{\text {nd }}$ Edition, 1997. 
[13] Baruchel, J., X-ray Tomography in Material Science, Hermes-Science, Paris, 2000.

[14] Kak, A.C. and Slaney, M., Principles of Computerized Tomographic Imaging, IEEE Press, New-York, 1988.

[15] Grangeat, P., La Tomographie, Hermes Science, Traité IC2, Paris, 2002.

[16] Cui, L. and Cahyadi, J.H., Permeability pore structure of OPC paste. Cement and Concrete Research, 31, pp. 277-282, 2001.

[17] Mindess, S., Young, J.F. and Darwin, D., Concrete $2^{\text {nd }}$ Ed., Englewood Cliffs (NJ): Prentice Hall, 2002.

[18] Pipilikaki, P. and Beazi-Katsioti, M., The assessment of porosity and pore size distribution of limestone Portland cement pastes. Construction and Building Materials, 23, pp. 1966-1970, 2009. 